\title{
La interpelación del presidente del Banco Central de Reserva
}

Con los resultados de las últimas elecciones legislativas y municipales se suscitó un importante cambio en la correlación de fuerzas políticas, tanto a nivel de los gobiernos locales como de la asamblea legislativa. Este elemento, catalizado por el multimillonario fraude financiero cometido en las instituciones FINSEPRO e INSEPRO, ha dado paso a iniciativas para dirimir responsabilidades nunca vistas en la historia reciente del país; aun cuando existen antecedentes de escándalos financieros previos, aunque de menor envergadura, como el de FOMIEXPORT en 1992 y CREDICLUB sólo unos meses antes de FINSEPRO-INSEPRO.

Una de las facetas más novedosas de esta nueva etapa del país ha sido la interpelación del Presidente del Banco Central de Reserva (BCR), Roberto Orellana Milla, sobre su actuación en el caso FINSEPRO-INSEPRO y, en alguna medida, sobre algunos aspectos relacionados con las características de! sistema financiero. Esta interpelación, y otros datos que más adelante se presentan, dieron lugar a que la asamblea legislativa emitiera una recomendación que sugería al Presidente de la República destituir al presidente del BCR de su cargo, por considerar que su acluación fue negligente frente al caso de defraudación mencionado. A contrapelo, el Presidente de la República decidió, apoyado por su Consejo de Ministros, ignorar la recomendación del pleno legislativo y ratificar en su cargo a Orellana Milla.

Los hechos presentados sugieren que la interpelación sólo fue una futilidad, pues no pasó de ser un simulacro de democracia, sin embargo, de su examen pueden surgir valoraciones de importancia sobre la conducción de la política económica y las transformaciones del país operados durante esta década.

El principal objetivo del cuestionario que guió la citada interpelación era explorar las relaciones de Orellana Milla con el sistema financiero, con posibles actos de tráfico de influencias y con el fraude FINSEPRO-INSEPRO. Los aspectos vinculados a los resultados de la política monetaria y crediticia sobre la estabilidad macroeconómica -que son competencia del Banco Central de Reserva - no fueron abordados. Lo anterior no es de extrañar, considerando que la interpelación surgió como resultado directo del escándalo financiero citado, y no como resultado de un cuestionamiento omnicomplexivo sobre el papel del banco en la economía. La exploración de la función del BCR en la estabilización macroeconómica apareció más como un tema aislado, sin mayor trascendencia para los legisladores, cuando en realidad debió ser el tema principal de la interpelación.

En lo que sigue, se consigna una pequeña reseña de la interpelación en lo tocante a los pocos aspectos técnico-económicos que se abordaron y al caso FINSEPRO e INSEPRO, para luego analizar datos que fueron aportados posteriormente y que influyeron de manera decisiva en el dictamen de la asamblea legislativa. Finalmente, se presentan algunas de las implicaciones de la interpelación y sus resultados.

Los aspectos técnico-económicos de la interpelación fueron en realidad muy pocos y, como 
se mencionó, ignoraron importantes aspectos relacionados con la política monetario-financiera desarrollada desde 1989, año en que asumió el cargo su actual presidente. Algunos de estos aspectos, abordados en el cuestionario inicial de 40 preguntas utilizado para la interpelación, son los relacionados con la concentración de las utilidades y los depósitos en sólo tres bancos del sistema y el manejo del encaje legal de FINSEPRO e INSEPRO. Más adelante, en la fase de repreguntas sobre el mismo cuestionario, se tocaron temas como la situación de las reservas de oro y las funciones específicas del Banco Central de Reserva, que no trascendieron más allá de su especificidad.

En lo que se refiere a la concentración de las utilidades, Orellana Milla afirmó que "No es nada particular de El Salvador, pues esto es normal en muchos sistemas financicros del mundo...que muestran una concentración, digamos que, bastante típica en los sistemas linancieros y en muchas otras actividades de otros sectores". No obstante, afirmó que en realidad sí existe competencia tanto de parte de las instituciones financieras internacionales como de la Bolsa de Valores local. Señaló, además, que "estamos con un sistema financiero y con instituciones bastante nuevas, entonces es lógico que el grado de competencia, eficiencia, competitividad del sistema se vaya desarrollando gradualmente. Existe un marco legal que es adecuado y que más bien permite, facilita, se excita la mayor competencia posible $y$ son marcos legales que atraen y permiten atraer inversión extranjera en el sector financiero para, precisamente, crear más competencia".

En este punto es necesario mencionar que, independientemente de lo que ocurre en otros países y de lo que el marco legal facilita, el nivel de concentración presente en el sistema financiero contradice de manera flagrante el espíritu de la liberalización económica, que considera el libre mercado como el mejor asignador de recursos, promotor de mayores niveles de eficiencia y un mecanismo ideal para la formación de precios relativamente bajos. La liberalización del sistema financiero -que en realidad no depende sólo del Banco Central de Reserva- ha producido una concentración de la actividad financiera en pocas manos y un sistema de asignación de tasas de interés activas artificialmente altas que, a su vez, multiplican los márgenes de utilidad bruta de los bancos.

La posibilidad de que en otros países exista concentración económica, no justifica que aquí también exista. Es justo reconocer que este resultado ha sido producto no sólo de la política desarrollada por el Banco Central de Reserva, sino de los proyectos de liberalización económica, especialmente la privatización de la banca, que fueron diseñados e implementados por el partido Alianza Republicana Nacionalista (ARENA); el cual llevó a Orellana Milla a la presidencia del BCR y fue el único partido político que abanderó incondicionalmente su defensa. La concentración de la banca es fruto de todo un plan de gobierno y no sólo del accionar de este banco.

En relación con el manejo del encaje legal de FINSEPRO, los diputados cuestionaron acerca del monto que fue utilizado en la ventanilla de liquidez automática, por considerar que ello podía haber alertado tempranamente sobre una inminente crisis financiera. Orellana Milla aprovechó la oportunidad para explicar lo que es el encaje legal y la situación del correspondiente a FINSEPRO, antes de que se volviera pública la defraudación. Según él, "Hasta el viernes 27 de junio...FINSEPRO siempre mantuvo el cumplimiento de este requisito del encaje". Sobre el uso de la ventanilla de liquidez automática, Orellana Milla expresó que estuvo "de acuerdo a un uso que era normal y de acuerdo con las normas y las leyes que regula el Banco Central".

Por otra parte, y como se mencionó antes, la fase de repreguntas colocó sobre el tapete otras situaciones más diversas que en la mayoría de casos tenía más relación con aspectos técnico-jurídicos e, inclusive, con situaciones personales que, hipotéticamente, inhabilitarían a Orellana Milla para desempeñar su cargo. Al final, estos elementos tuvieron poco peso para formar criterio en los diputados.

Lo que destaca es que en prácticamente todos los cuestionamientos, Orellana Milla se eximió de culpabilidad en el caso FINSEPRO e INSEPRO, alegando que la fiscalización de instituciones financieras no es competencia del Banco Central de Reserva. La institución encargada sería la Superintendencia del Sistema Financiero, que no actuó oportunamente y sería la responsable de que el fraude no haya 


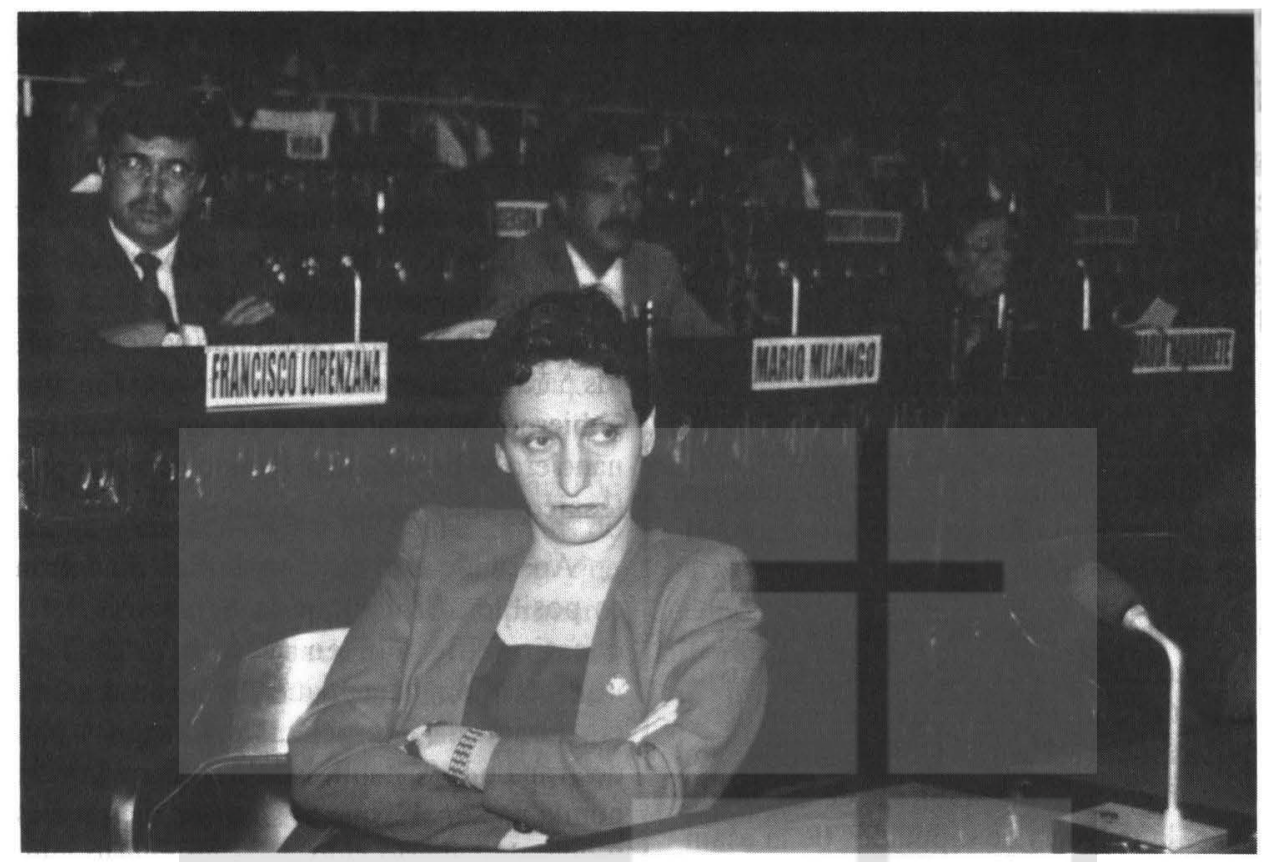

podido prevenirse. Según Orellana Milla, el artículo 3 de la Ley Orgánica del BCR define como su función primordial "velar por la estabilidad de la moneda y...promover y mantener las condiciones monetarias, cambiarias, credilicias y financieras más favorables para la estabilidad de la cconomía nacional". Por tanto, según el razonamiento de Orellana Milla no sería compelencia del Banco Central de Reserva la fiscalización de empresas financieras ni, mucho menos, actuar en caso que se sospechen posibles violaciones al marco legal o de defraudación a la economía pública. En un primer momento, este planteamiento pareció convencer a los diputados de oposición de que su actuación en el caso FINSEPROINSEPRO estuvo apegada a la ley y hasta a la ética profesional. Incluso, fuentes vinculadas a la asamblea legislativa, anticipaban que no se obtendría la votación necesaria para recomendar al Presidente de la República su destitución.

Como se verá más adelante, fue la comparecencia del ex Superintendente, Rodríguez Loucel, ante los diputados de la asamblea legislativa lo que cambio radicalmente la correlación de fuerzas, y dio paso a la posterior recomendación de destituir a Orellana Milla.

La única fracción legislativa que se pronunciaba abiertamente en favor de la destitución de Orellana
Milla era la del Frente Farabundo Martí para la $\mathrm{Li}$ beración Nacional (FMLN). Por su parte, ARENA mantenía su postura de apoyo hacia el presidente del BCR. La decisión final dependía fundamentalmente de la postura de los partidos minoritarios como el Partido de Conciliación Nacional (PCN) y el Partido Demócrata Cristiano (PDC) los cuales, en un primer momento, parecían más inclinados a favor de la no destitución de Orellana Milla.

En este mismo contexto, la Asociación Nacional de la Empresa Privada (ANEP) y la Asociación Bancaria Salvadoreña (ABANSA), también expresaron su oposición a un dictamen desfavorable para Orellana Milla, por considerar que éste había defendido técnicamente su postura y que ha sido el artífice de la actual situación de estabilidad macroeconómica del país. Más tarde, ABANSA accedería a aportar los fondos para un fideicomiso destinada a cancelar parte de lo estafado. Según las posturas de estas gremiales, la destitución de Orellana Milla sólo cabe dentro de una estrategia política y podría llegar a comprometer la estabilidad del país.

Vale la pena detenerse aquí para señalar que esta postura, pese a pretender ser un llamado a la nopolitización, en realidad sugiere que las hipótesis acerca de la vinculación orgánica entre el partido 
ARENA y la mayoría del sector empresarial del país son verdaderas. Pretender atribuir al presidente del BCR el "mérito" de la situación económica del país es un argumento especioso e ideologizado, con la clara intencionalidad de dar un espaldarazo al partido de gobierno en uno de sus momentos más críticos. La estabilidad económica del país proviene fundamentalmente del flujo de remesas familiares, son éstas las que permiten acumular reservas internacionales netas - pese al enfrentamiento de constantes déficits en la balanza comercial-, mantener estable el tipo de cambio, estabilizar los precios internos, incrementar el consumo y la producción -especialmente del sector terciario-, etc.

Después de las declaraciones vertidas por el ex Superintendente Rodríguez Loucel ante la Comisión Especial Investigadora de Actividades Financieras, las perspectivas sobre el caso de Orellana Milla cambiaron drásticamente. Al día siguiente de las citadas declaraciones, la mayoría de diputados de la asamblea legislativa aprobó recomendar al Presidente Calderón Sol destituirlo de su cargo. Según las declaraciones del ex Superintendente, desde el mes de enero pasado hasta el 15 de mayo último, él habría sostenido reuniones con diferentes personeros del gobierno -incluidos los presidentes de la república y del BCR - en las que se habría tratado la situación de FINSEPRO e INSEPRO en, por lo menos, 10 ocasiones. Esto resultó grave para el presidente del BCR, pues éste afirmó ante el pleno de la asamblea legislativa haber conocido las irregularidades del caso INSEPRO apenas en junio pasado.

Después de la lectura de estos datos, resulta claro que alrededor del caso de FINSEPRO e INSEPRO no se encuentra sólo la obra de empresarios estafadores, sino que también se perciben implicaciones más profundas con la sociedad política y otros miembros del sector empresarial. Por una parte, las reuniones reseñadas por Rodríguez Loucel, sostenidas por el Presidente de la República, uno de los ofendidos por los directivos de FINSEPRO -el Canciller de la República Ramón González Giner-, y los encargados de velar por la estabilidad y transparencia del sistema financiero, sugieren que en realidad no se pretendía actuar en contra de los otrora grandes contribuyentes para la causa de ARENA, ahora catalogados como estafadores. Cabe destacar aquí que
Roberto Mathies Hill, el principal implicado en el fraude financiero, se desempeñaba como Presidente del Sector Empresarial de ARENA hasta antes de su captura por defraudación de la economía pública.

Esta situación da lugar a concluir que sí existió tráfico de influencias, tanto por pretender favorecer a los implicados dándoles tiempo para resolver su situación financiera, como por pretender presionarlos para saldar cuentas con otro alto funcionario del gobierno. Es notable que el manejo del sistema financiero se ha venido haciendo no sólo con criterios técnicos, sino también políticos.

Aparte de este caso específico de defraudación, es posible afirmar que la actuación del BCR es cuestionable aún en el ámbito de la gestión macroeconómica, aunque ello no haya salido a relucir durante la interpelación. Existen aspectos que derivan de la "estabilidad de la moneda y de la economía nacional", que ni siquiera fueron explorados y que, de haberlo sido, habrían puesto aún más en entredicho la eficiencia del Banco Central de Reserva (BCR) en el desempeño de sus funciones principales. Según versiones difundidas por el mismo banco, los principales resultados de su gestión han sido revertir el estancamiento económico de la década de los ochenta, haber provocado una reducción de la inflación, la estabilización en el tipo de cambio, el incremento de las Reservas Internacionales Netas (RIN) y el mantenimiento las tasas de interés libres y competitivas.

Esto es, en realidad, una verdad a medias pues, como se mencionó antes, las condiciones para el crecimiento y la estabilidad económica han sido determinadas por procesos que escapan completamente del control de dicho banco. La inyección de recursos externos (empréstitos, donaciones y especialmente remesas) ha tomado mayor auge durante esta década y explican fundamentalmente el comportamiento de la economía. La tasa de interés es la variable sobre la cual este banco puede tener mayor incidencia y, paradójicamente, persisten dudas razonables sobre su nivel excesivamente alto, así como de las supuestas bondades de su liberalización.

La interpelación del presidente del BCR obvió aspectos fundamentales que debieron ser interrogados y que cuestionan su actuación como, por ejem- 
plo, cuál ha sido su función en la estabilización económica (tomando en cuenta lo ventajoso que resulta contar con llujos externos de capital), en el actual proceso de recesión económica que ya se prolonga por más de dos años, en la concentración del crédito en actividades terciarias, en el desfase de las tasas de interés en relación con la inflación y en la manipulación de aquellas para incrementar las ganancias de las instituciones financieras. Estos factores inciden, directa e indirectamente, en la estabilidad de la economía nacional.

Aun con el deficiente diseño del cuestionario de interpelación, y sin considerar las declaraciones de Rodríguez Loucel, resultan claros dos aspectos que cuestionan la actuación de Orellana Milla: primero, que ignoró señales tempranas de alerta sobre el caso $\mathrm{y}$, segundo, que responde a los intereses del sector empresarial - especialmente el financiero-y de los funcionarios de gobierno, más que a una legítima búsqueda de la estabilidad macroeconómica.

El éxito de su gestión al frente del Banco Central de Reserva no es por méritos propios. El banco se encuentra en una posición sumamente cómoda debido a la estabilidad macroeconómica que deriva de la gigantesca afluencia de remesas familiares. El BCR se limita a administrar la solvencia que otorgan las remesas.

No es ideologizada la afirmación de que la ratificación de Orellana Milla en su cargo, no implica que su manejo del caso FINSEPRO-INSEPRO, y de la política monetario-crediticia, sea el más adecuado para la problemática del país. Únicamente implica que, a nivel del ejecutivo, se han cerrado filas ante posibles críticas a su gestión provenientes, ya sea de la asamblea legislativa o de la misma sociedad civil. En realidad, el trabajo del Banco Central de Reserva se enmarca en todo un proyecto de nación que ha venido implementándose desde 1989 y, por ello, su cuestionamiento atañe al gobierno en general.
Una de las lecciones más importantes que deben retomarse de la citada interpelación sería que las futuras interpelaciones no se diseñen en función de fines políticos, para el caso, la implicación de Orellana Milla en el caso FINSEPRO-INSEPRO y en posibles violaciones a la Ley Orgánica del $\mathrm{BCR}$ como argumentos para proponer su destitución, sino más bien en función de cuestionar y mejorar el desempeño de los funcionarios de Estado en sus áreas de competencia, ya sea con un mejor desempeño de sus funciones o con una modificación del marco jurídicoinstitucional que regula sus actuaciones.

La situación actual llama la atención sobre las características negativas del modelo de crecimiento económico configurado durante los últimos años. La liberalización de la economía se está traduciendo en una proliferación del sector terciario, especialmente del financiero, que opera con la libertad suficiente como para incurrir en defraudaciones de la economía pública.

La interpelación a Orellana Milla muestra, por un lado, cómo se ha instrumentalizado una situación económica bonancible - la afluencia de remesas familiares - para beneficiar la imagen del Banco Central de Reserva, llegando incluso a presentarse como el artífice de la relativa estabilidad económica. Por otro lado, al considerar las argumentaciones presentadas sobre la concentración económica en la banca, llama la atención el hecho de que el gobierno la justifica y, a la vez, genera las condiciones para privilegiar la actividad del sector financiero. No es casual que los empresarios más favorecidos hayan otorgado apoyo institucional y financiero al residente del Banco Central de Reserva, pues el cuestionamiento de su actuación también era un cuestionamiento al modelo económico del cual se están sirviendo.

Luis E. Romano 